\title{
Correspondence
}

\section{Tracheal compression as a cause of respiratory symptoms after repair of oesophageal atresia}

\section{Sir,}

The article by Cook and Bush (Archives, 1978, 53, 246) draws attention to tracheal compression in infants with oesophageal atresia as a cause of respiratory symptoms and recurrent chest infections. In their case, the compression was due to pulsation of the aortic arch and was relieved by tracheopexy. We fully agree that careful bronchoscopic and oesophagoscopic investigations should be performed if signs of tracheal compression are observed.

In our series of 117 cases of oesophageal atresia (1965-77), there was evidence of tracheal compression or tracheomalacia in 27: in 7, there was a congenital cardiovascular lesion (in 4, this included abnormal origin of arterial brachiocephalic truncus); in 9, a gross subanastomotic dilatation of the oesophagus (Figs 1 and 2)

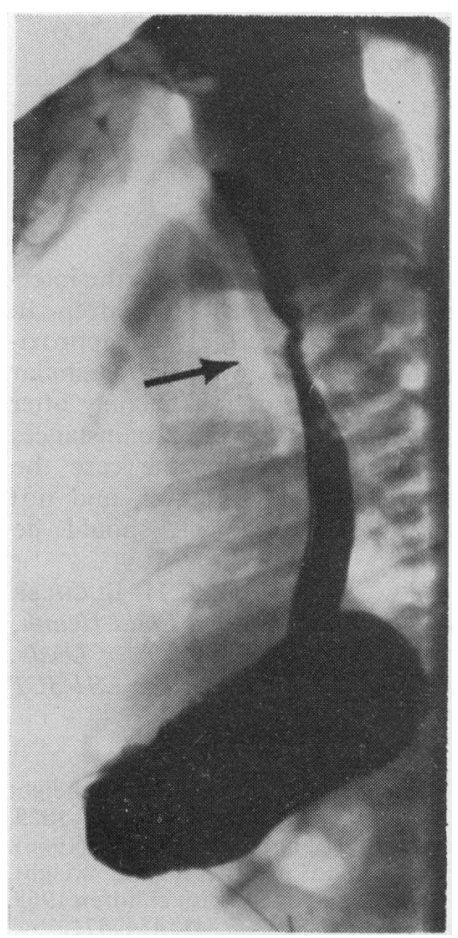

Fig. 1 Normal trachea after oesophageal anastomosis (arrow).

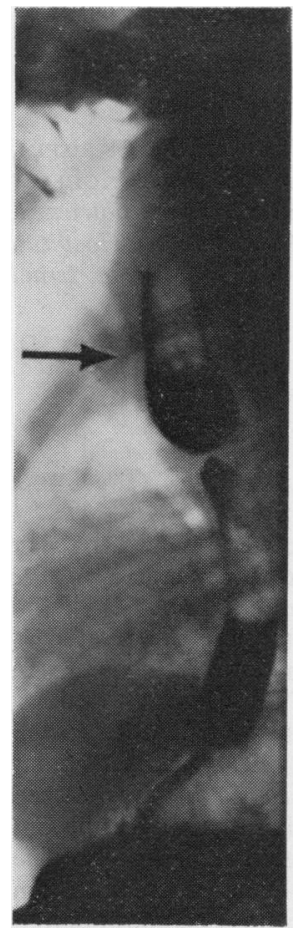

Fig. 2 Flattened lumen of trachea due to gross subanastomotic dilatation of oesophagus, 5 weeks later (arrow).

cured by progressive bougienage; in 2 , both these two causes; in 9, no definite compression but a tracheomalacia with inspiratory flattening of lumen. Repeated milk inhalation may also contribute to respiratory infection, while tracheopexy is helpful in vascular compressions; in 'idiopathic' tracheomalacia no treatment seems to be available, and apnoeic and cyanotic attacks together with inhalation pneumonia remain a permanent hazard.

R. Caldera, J. P. Chabrolle, and A. Rossier Hôpital Saint-Vincent-de-Paul, 74 Avenue Denfert-Rochereau, 75674 Paris Cedex 14, France

\section{Cystinotic rickets treated with vitamin D metabolites}

Sir,

With reference to the article by Etches et al. (Archives, $1977,52,661$ ), we should like to add two further cases which we have treated successfully with $1-\alpha$-hydroxycholecalciferol. 
Both children, a brother and sister, had typical cystinosis, severe rickets, and advanced renal failure. Both had previously been unsuccessfully treated with high doses of vitamin D2, with calcium and phosphorus supplements. We found, in contradistinction to Etches et al., that with our treatment in both cases serum calcium was raised, while phosphorus fell almost to normal level and with little fluctuation. There was clinical improvement, bone pains disappeared in 8 days, and radiological healing was complete in $3 \frac{1}{2}$ months.

Siegfried I. Beer, Ellis VURe, Chaim Krispin, MORDECHAI WARON, and DAVID KOHELET Department of Pediatrics $B$, Assaf Harofeh Hospital, Zerifin, Israel

\section{Variations in height throughout the day}

Sir,

It is well known that stature becomes less as the day progresses, but it is usually considered that gentle traction upwards on the head when measuring height will compensate for this effect. Whitehouse et al. (1974), using this technique, showed an average decrease in stature from 9.30 a.m. to 2 p.m. of only $2 \mathrm{~mm}$ in 19 boys, and from 10 a.m. to 5 p.m. of $4.6 \mathrm{~mm}$ in another group of 11 boys. Strickland and Shearin (1972), using a similar technique, showed a mean difference of $1.54 \mathrm{~cm}$ between the height of 100 children immediately after getting out of bed in the morning and between 4 and 5 p.m. The significance of the difference of heights dependent on time of day of measurement has recently become apparent in a longitudinal growth study. Estimations of the height of a subject undertaken in the latter part of the day using the method described by Tanner et al. (1966) with gentle traction have shown significant differences from those performed in the morning by the same technique and by the same competent observer (who was not influenced by knowledge of previous measurements).

28 boys (age range 7-14 years) have been measured in this way between 6 and 9 p.m. and between 9 a.m. and noon the following morning. Except for 2 subjects for whom the two measurements were the same, the morning height was greater than the evening one by amounts ranging from 0.1 to $1.4 \mathrm{~cm}$ (mean of 0.6 ). The bulk of this change is in the trunk, as expected, and was indicated by similar changes in sitting height to those of total stature.

Eight boys were measured late one evening and repeatedly through the ensuing day. Their measurements are shown in the Figure. The difference between the maximum and minimum heights for any individual is considerable (the maximum always being that on first rising in the morning), ranging from 0.8 to $2.8 \mathrm{~cm}$. A large proportion of the extra early morning height is lost within the first few hours of being mobile during the day. The decline in height is not always continuous throughout the day nor are measurements necessarily identical at similar times on consecutive days. This may imply tha even within the course of a day the pattern of activity? may affect stature to a small degree.

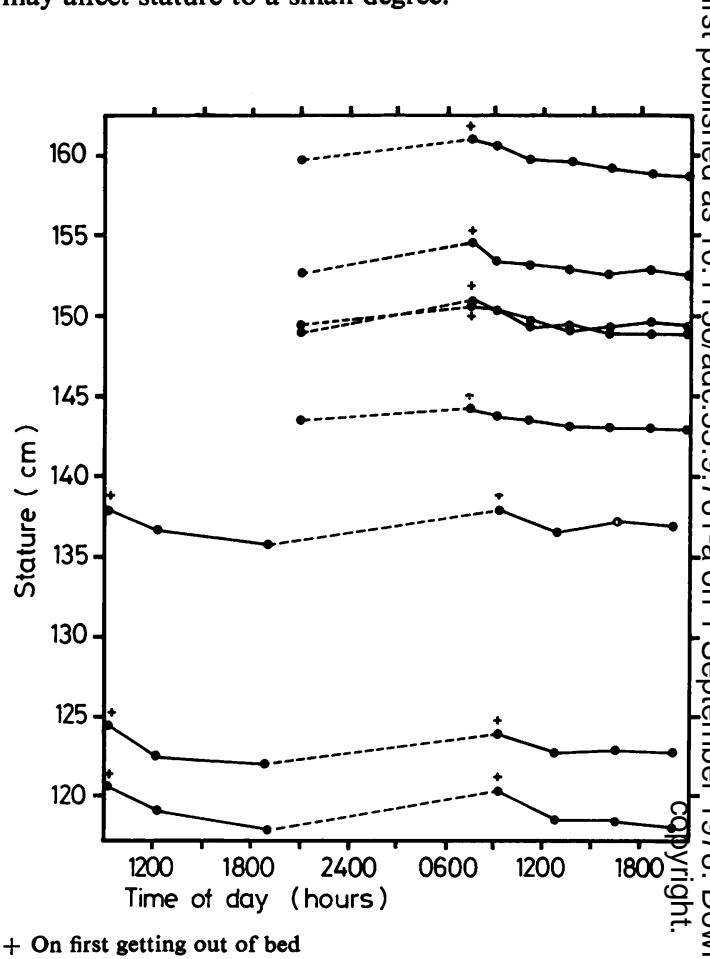

Figure Measurement of stature of 8 boys at various times on two consecutive days.

These observations must have implications in the inter $\frac{}{3}$ pretation of serial height measurements of children. In theory, observations should be undertaken at approxi-f mately the same time of day and following a similar pattern of activity, but certainly not immediately aftero getting out of bed. In the clinic these circumstances? frequently apply, but when this is not the case the possibility of an error due to this variation, and no necessarily to accuracy of measurement, should beo considered.

J. M. H. BUCKLER
Department of Paediatrics and Child Health,
University of Leeds?
27 Blundell Street, Leeds LS1 3ET
S

References

Strickland, A. L., and Shearin, R. B. (1972). Diurnal heighN variation in children. Journal of Pediatrics, 80, 1023-1025.

Tanner, J. M., Whitehouse, R. H., and Takaishi, M. (1966) Standards from birth to maturity for height, weighto height velocity, and weight velocity: British children 1965 Part I. Archives of Disease in Childhood, 41, 454-471.

Whitehouse, R. H., Tanner, J. M., and Healy, M. J. R! (1974). Diurnal variation in stature and sitting height in 12-14 year old boys. Annals of Human Biology, 1, 103-106유 